\title{
Social learning of arbitrary food preferences in bonobos
}

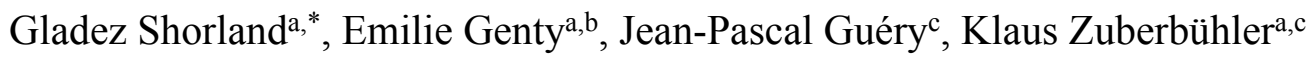

a Department of Comparative Cognition, Institute of Biology, University of Neuchâtel, Rue Emile-Argand 11, 2000 Neuchâtel, Switzerland.

b Institute of Work and Organizational Psychology, University of Neuchâtel, Rue EmileArgand 11, 2000 Neuchâtel, Switzerland.

c La Vallée des Singes Primate park, Le Gureau, 86700 Romagne, France.

d School of Psychology and Neurosciences, University of St Andrews, St Mary's Quad, South Street, St Andrews, KY16 9JP, Scotland, UK.

E-mail: E. Genty, emilie.genty@unine.ch; J-P. Guéry, jp.guery@la-vallee-des-singes.fr; K. Zuberbühler, klaus.zuberbuehler@unine.ch.

Corresponding author:

G. Shorland

gladez.shorland@,outlook.com

Department of Comparative Cognition, Institute of Biology, University of Neuchâtel, Rue Emile-Argand 11, 2000 Neuchâtel, Switzerland.

* Correspondence: G. Shorland, Department of Comparative Cognition, Institute of Biology, University of Neuchâtel, Rue Emile-Argand 11, 2000 Neuchâtel, Switzerland. e-mail address: gladez.shorland@outlook.com 


\section{Highlights}

- Bonobos socially learn and copy the arbitrary food preferences of others.

- Bonobos copied others' arbitrary preferences of novel foods on first attempts.

- Subject age and exposure time have a positive effect on successful social learning.

- Copied food preferences can remain stable despite having better knowledge. 


\section{ABSTRACT}

A fruitful approach to investigate social learning in animals is based on paradigms involving the manipulation of artefacts. However, tool use and elaborate object manipulations are rare in natural conditions, suggesting that social learning evolved in other contexts where fitness consequences are higher, such as discriminating palatable from noxious foods, recognising predators or understanding social hierarchies. We focussed on one such context by investigating whether bonobos socially learned others' arbitrary food preferences through mere observation. To this end, we trained two demonstrators to prefer or avoid distinctly coloured food items, treated with either a sweet or bitter agent. Demonstrators then displayed their newly acquired preferences in front of naïve subjects. In subsequent choice tests, subjects generally matched their choices to the demonstrators' preferred food colours, despite having already tasted the equally palatable colour alternative. Both age and exposure to demonstrator preference had a significant positive effect on the proportion of matched choices. Moreover, in a context where errors can be costly, social learning was instant insofar as six of seven subjects used socially learned information to influence their very first food choice. We discuss these findings in light of the current debate on the evolution of social learning in animals. 


\section{Social learning of arbitrary food preferences in bonobos}

2 Gladez Shorland ${ }^{a^{*}}$, Emilie Genty ${ }^{\mathrm{a}, \mathrm{b}}$, Jean-Pascal Guéryc, Klaus Zuberbühler ${ }^{\mathrm{a}, \mathrm{d}}$

4 a Department of Comparative Cognition, Institute of Biology, University of Neuchâtel, Rue 5 Emile-Argand 11, 2000 Neuchâtel, Switzerland.

6 b Institute of Work and Organizational Psychology, University of Neuchâtel, Rue Emile7 Argand 11, 2000 Neuchâtel, Switzerland.

$8 \quad{ }^{\mathrm{c}}$ La Vallée des Singes Primate park, Le Gureau, 86700, Romagne, France.

9 d School of Psychology and Neurosciences, University of St Andrews, St Mary's Quad, South 10 Street, St Andrews, KY16 9JP, UK.

12 E-mail: E. Genty, emilie.genty@unine.ch ; J-P. Guéry, jp.guery@la-vallee-des-singes.fr; K.

13 Zuberbühler, klaus.zuberbuehler@unine.ch

* Corresponding author:

16 G. Shorland

17 e-mail: gladez.shorland@outlook.com

18 Address: Department of Comparative Cognition, Institute of Biology, University of

19 Neuchâtel, Rue Emile-Argand 11, 2000 Neuchâtel, Switzerland.

\section{ABSTRACT}

A fruitful approach to investigate social learning in animals is based on paradigms involving the manipulation of artefacts. However, tool use and elaborate object manipulations are rare in natural conditions, suggesting that social learning evolved in other contexts where fitness consequences are higher, such as discriminating palatable from noxious foods, recognising predators or understanding social hierarchies. We focussed on one such context by investigating whether bonobos socially learned others' arbitrary food preferences through mere observation. To this end, we trained two demonstrators to prefer or avoid distinctly 
coloured food items, treated with either a sweet or bitter agent. Demonstrators then displayed their newly acquired preferences in front of naïve subjects. In subsequent choice tests, subjects generally matched their choices to the demonstrators' preferred food colours, despite having already tasted the equally palatable colour alternative. Both age and exposure to demonstrator preference had a significant positive effect on the proportion of matched choices. Moreover, in a context where errors can be costly, social learning was instant insofar as six of seven subjects used socially learned information to influence their very first food choice. We discuss these findings in light of the current debate on the evolution of social learning in animals.

Keywords: cognition; foraging; nonhuman primate; novel food; Pan paniscus; social learning

\section{Introduction}

Over the past decades, social learning has become a major topic in the field of comparative cognition (Whiten and van de Waal, 2018). Social learning, or more specifically "learning that is influenced by observation of, or interaction with, another animal (typically a conspecific) or its products" (Heyes, 1994) can be highly adaptive as it allows individuals to avoid costly trial-and-error learning, saving both time and energy and avoiding dangerous mistakes (Hopper et al., 2011). Furthermore, social learning can favour the rapid spread of advantageous behavioural innovations and, as such, acts as a 'second inheritance system' in addition to phylogenetically acquired behavioural traits (Whiten, 2005). Social learning is likely to be adaptive in many domains, including foraging, mate choice or predator avoidance (Galef and Giraldeau, 2001; Galef and Laland, 2005) and can even take place between species. Indeed, within a given ecological niche, different species are likely to be faced with the same requirements and constraints, as such, information acquired from heterospecifics 
can be just as valuable as that acquired from conspecifics (Avarguès-Weber et al., 2013). For example, the existing literature documents cases of interspecific social learning of food source location in insects (Dawson and Chittka, 2012) and fish (Coolen et al., 2003), of predator recognition and avoidance in mammals (Kitchen et al., 2010; Zuberbühler, 2000) and reptiles (Vitousek et al., 2007) and of nesting site preference in birds (Seppänen and Forsman, 2007). The importance of social learning is particularly relevant for young and naïve individuals, who can avoid costly or maladaptive behaviour by observing and learning from more experienced and older individuals that have, essentially, survived to adulthood in a given environment (Galef and Laland, 2005).

Over the years, substantial efforts have been made to investigate the mechanisms underlying social learning across different groups of animals, using both observational and experimental techniques in the wild (e.g. apes: Hobaiter et al., 2014; monkeys: Kawai, 1965; birds: Aplin et al., 2014) and in captivity (e.g. apes: Clay and Tennie, 2018; Dindo et al., 2011; Whiten et al., 2005; monkeys: Dindo et al., 2008; van de Waal et al., 2013b; lemurs: Stoinski et al., 2011; birds: Auersperg et al., 2014; see Reader and Biro, 2010 for a non-exhaustive survey). A common approach to study social learning processes has been to use paradigms that require manipulation of a container ('puzzle box') to extract an edible reward. A particularly successful variant is the 'artificial fruit' (Whiten et al., 1996), which can be opened through various means (e.g. lifting or sliding a door) to obtain a reward within. Such experiments have brought to light not only evidence of social learning (e.g. van de Waal et al., 2010, 2013b) and social diffusion (e.g. Dindo et al., 2008, 2011; Whiten and Mesoudi, 2008) but also of social conformity in animals (e.g. Dindo et al., 2009; Whiten et al., 2005). Overall, these findings have broad implications for theories of the evolution of culture (see Whiten 
and van de Waal, 2016a for a review) as they begin to unravel the basic building blocks for the human capacity for culture, many of which are shared with non-human primates.

Another particularly common experimental paradigm in the laboratory is to expose subjects to inaccessible food rewards that can only be accessed using a tool (e.g. Call and Tomasello, 1994; Horner and Whiten, 2005). However, while tool use is taxonomically widespread, it is generally rare in most primate and non-primate species (Hunt et al., 2013), raising questions on the extent to which experimental paradigms based on tool use are adequate to investigate social learning capacities in such species. Most primates are capable of rich arrays of manipulations, such as twisting, pulling, or peeling, but these manipulations tend to be structurally simple (but see Byrne et al., 2001) and rarely involve tool use. Moreover, although some chimpanzee communities have well established tool traditions (Visalberghi et al., 2015) others only rarely use tools (Lamon et al., 2017; Lamon and Zuberbühler, 2015; Reynolds, 2005), and tool use is curiously absent in wild bonobos (Koops et al., 2015) and many other primate species. However, although social learning may give naïve observers an advantage in some types of problem solving such as moss-sponging in chimpanzees (Hobaiter et al., 2014) and nut-cracking in tufted capuchins (Coelho et al., 2015) and chimpanzees (Marshall-Pescini and Whiten, 2008), and although this capacity for tool use may translate into fitness consequences in certain contexts, for example, by providing highly nutritional foods when usual food resources are scarce (Yamakoshi, 1998), tool use may not

97 be as significant for survival as general foraging strategies.

Social learning, in other words, may be better investigated in relation to more universally ecologically relevant challenges, such as learning how to interact with socially powerful group members or neighbouring groups or learning how to identify animal species that can 
102 pose a predatory threat. Indeed, a recent study has revealed that chimpanzees learn rapidly

103 from each other how to react to humans, a major predator of chimpanzees (Samuni et al.,

104 2014). Regarding more ecologically relevant generalised foraging strategies, one of the key

105 domains for social learning is to discriminate edible from noxious foods (van de Waal et al.,

$1062014,2013 a)$. Although neophobia is clearly adaptive when encountering novel foods

107 (Addessi et al., 2005), it exposes individuals to starvation when environmental conditions

108 become unstable. A more adaptive strategy may be to follow a 'copy-when-uncertain'

109 strategy when relying on individual learning alone is risky (Laland, 2004). Evidence of social

110 learning of food choice has been brought to light in several bird species (e.g. house sparrows:

111 Fryday and Greig-Smith, 1994; red-winged blackbirds: Mason and Reidinger, 1981) but also

112 in primates, where food-related social learning is influenced by a number of factors. These

113 include sex, rank, age and association (Coussi-Korbel and Fragaszy, 1995) and while in some

114 species ingestion of novel foods is facilitated by the mere presence of conspecifics, regardless

115 of what they eat (e.g. tufted capuchin: Addessi and Visalberghi, 2001; chimpanzee: Finestone

116 et al., 2014), in others, individuals appear to learn something about the palatability of foods

117 by observing others (e.g. cotton-top tamarin: Snowdon and Boe, 2003; vervet monkey: van

118 de Waal et al., 2013a). Acquisition of such social information may be permitted by simple

119 social learning processes such as stimulus or local enhancement (Whiten and van de Waal, 120 2018).

122 One important factor in primate social learning of foraging behaviour is the attention that young and naïve individuals pay to adult behaviour (Rapaport and Brown, 2008). Immature individuals often wait for more experienced individuals to begin foraging before following suit with the same food (e.g. Tarnaud, 2004; Whitehead, 1986; reviewed by Rapaport and

126 Brown, 2008). In apes, adult chimpanzees show more caution and close observation of 
127 conspecific food-handling when presented with novel foods than familiar ones (Gustafsson et

128 al., 2014). Infant apes are very attentive to their mothers during foraging and show high rates

129 of co-feeding (Jaeggi et al., 2010; Rapaport and Brown, 2008; Ueno and Matsuzawa, 2005).

130 Food sharing and co-feeding between mother and offspring seem to provide infants with

131 direct learning opportunities and has been observed in lowland gorillas, orangutans,

132 chimpanzees and bonobos (see Rapaport and Brown, 2008 for review). Interestingly, this

133 effect can be so strong that in orangutans, for example, the diet differences between mothers

134 is larger than between mothers and their offspring (Jaeggi et al., 2010). Despite these

135 considerations, individual learning remains an important mechanism for acquiring food

136 aversion (e.g. pigtail macaques, spider monkeys, Fairbanks, 1975; tufted capuchins,

137 Visalberghi and Addessi, 2000) or complex forms of food processing (e.g. nettle feeding in

138 gorillas, Tennie et al., 2008). Some feeding behaviours are even thought to be part of species-

139 specific behavioural repertoires (e.g. rough-leaf swallowing in chimpanzees and bonobos,

140 Menzel et al., 2013) although social influences likely aid the spread of such behaviours

141 (Huffman and Hirata, 2004). Following an initial phase in which young primates

142 preferentially learn from their mothers, their pool of accessible information broadens as their

143 social learning strategies evolve and they begin to learn from other group members (Whiten

144 and van de Waal, 2018).

146 When these strategies lead to learning from other group members, then selectiveness for

147 specific models can become apparent. In a recent review, Whiten and van de Waal (2018)

148 presented four selective learning biases evidenced in primates when they learn from other

149 group members. Namely, bias for 'knowledgeable' or 'expert' models; for older models, for

150 models of a specific sex or for conformity (i.e., copying a behaviour expressed by a majority

151 of one's group). Indeed, a general finding in empirical work on social learning in primates 
highlights the importance of the seeding demonstrator's identity. Chimpanzees, for example,

153 show a clear bias for copying older, higher-ranking and more knowledgeable individuals

154 (Biro et al., 2003; Kendal et al., 2015). However, low ranking individuals may be just as

155 effective in seeding novel behaviours when there is no 'model competition' (i.e., when there

156 are no older or higher ranking individuals acting as demonstrator) (Watson et al., 2017). Yet,

157 in a recent field study with chimpanzees, a novel behaviour, 'moss-sponging' invented by the

158 alpha male, was shown to spread through the community via two transmission patterns.

159 Initial spreading was within a spatio-temporal, proximity-based cohort but then mainly

160 through the matrilines (Lamon et al., 2017), a pattern not previously reported in captive

161 groups. The study thus indicated considerable flexibility of behaviour transmission patterns

162 in chimpanzees. Indeed, in recent years, evidence for the flexible use of social learning

163 strategies (i.e., what, when and whom to copy) has been found in several species spanning

164 across taxa, from fish and birds to rats and primates (see Kendal et al., 2018; and Rendell et

165 al., 2011 for reviews). This flexible use of social learning strategies is reflected in changes

166 linked to ontogeny, experience, state and context (Kendal et al., 2018) and is likely an

167 important factor as individuals enter the third phase of social learning proposed by Whiten

168 and van de Waal (2018), which occurs when individuals migrate to new groups and are

169 confronted with new locations and populations. For example, in an experiment in the wild,

170 migrating male vervet monkeys abandoned their personal food preferences to adopt an

171 opposite preference shown by their new group, which has been interpreted as potent social

172 learning with a conformist bias (van de Waal et al., 2013a; Whiten and van de Waal, 2016b).

174 In the midst of these potential strategies for social learning, we were interested in

175 experimentally assessing social learning in bonobos, in a universally ecologically relevant

176 situation, i.e., food choice. In the present study we tested whether bonobos are able to 
177 socially learn the arbitrary food preferences of a group member acting as demonstrator

178 through mere observation and whether they would adopt and maintain such preference

179 regardless of their own knowledge that both options were equally palatable. To achieve this

180 we ran a social learning experiment comprising of a series of experimental blocks in which

181 subjects observed two demonstrators consistently choosing food items of one novel colour

182 over another. Subjects were then tested to find out whether they preferred to choose food of

183 the same colour as the demonstrator. We predicted that, if subjects observed demonstrators

184 exhibiting a clear choice bias for one novel food colour over another, they would match this

185 bias above chance level in subsequent choice tests. As subjects had no prior experience with

186 these artificially coloured foods, we predicted social learning to be particularly strong during

187 the first experimental block when subjects were still naïve relative to these foods. We were

188 also interested in whether subjects were prepared to maintain such socially learned food

189 preferences, even after having experienced the respective colour alternatives.

\section{2. Methods}

\subsection{Ethical Note}

192 The study was authorised and ethically approved by the management of "La Vallée des

193 Singes". Although two individuals were regularly isolated for short periods of time ( $<30 \mathrm{~min})$

194 during this study, they were specifically selected based on their propensity to choose isolation

195 from the group on occasion thus avoiding stress for both the isolated individuals and the

196 group as a whole. The keeper isolated the individuals using methods regularly used on the

197 group when encouraging individuals to pass from one cage to another. When stress was

198 detected within the group, testing was postponed. The study was in line with

199 recommendations in the ARRIVE guidelines, and Animal Behaviour (1992) as well as the

200 EAZA and the AFdPZ code of ethics. 


\subsection{Study Site and Subjects}

202 The experiment took place between February and November 2014 at La Vallée des Singes

203 primate park, Romagne (France). Study subjects were selected from a large group of captive-

204 born bonobos $(N=17,8$ males and 9 females, age range: 14 months -45 years, mean $=15.2$

205 years, see table 1), housed in a large indoor enclosure $\left(400 \mathrm{~m}^{2}\right)$ with access to two outdoor

206 wooded islands covering $11,500 \mathrm{~m}^{2}$ in total.

207

208 Table 1. Study subjects housed at La Vallée des Singes and role in the experiment

\begin{tabular}{llllll}
\hline \multicolumn{2}{l}{ Individual } & Sex & Birth year & Age-class & Role \\
\hline Diwani & $(\mathrm{DW})$ & $\mathrm{M}$ & 1996 & Adult & Demonstrator \\
Kelele & $(\mathrm{KEL})^{*}$ & $\mathrm{M}$ & 2004 & Adult & Demonstrator \\
\hline Ulindi & $(\mathrm{UL})$ & $\mathrm{F}$ & 1993 & Adult & Observer \\
Lingala & $(\mathrm{LNG})$ & $\mathrm{F}$ & 2003 & Sub-adult & Observer \\
Lucy & $(\mathrm{LY})$ & $\mathrm{F}$ & 2003 & Sub-adult & Observer \\
Nakala & $(\mathrm{NK})^{*}$ & $\mathrm{~F}$ & 2007 & Juvenile & Observer \\
Loto & $(\mathrm{LO})$ & $\mathrm{M}$ & 2009 & Juvenile & Observer \\
Moko & $(\mathrm{MO})$ & $\mathrm{M}$ & 2012 & Infant & Observer \\
Khalessi & $(\mathrm{KLS})$ & $\mathrm{F}$ & 2012 & Infant & Observer \\
\hline
\end{tabular}

Individuals marked by an asterisk had the same father; age-class as defined by Kano (1984)

210

211 Two adult males, DW and KEL, were selected as demonstrators based on the ease with which

212 they could be isolated without apparent signs of stress to any of the group members. Six

213 experimental blocks were carried out, each consisting of a Demonstrator Training phase

214 (DT) for the demonstrators, followed by a Preference Demonstration phase (PD) in front of

215 the subjects and finally an Observer Testing phase (OT) in which subjects underwent

216 repeated choice tests. Three of the six experimental blocks were in the pink condition (i.e.,

217 demonstrator preference was for pink courgette) and three in the blue condition (i.e., 
218 demonstrator preference was for blue egg, further details are specified below). Testing was

219 dependent on the personal motivation of each individual to, first, observe the Preference

220 Demonstrations (PD), and, second, participate in the food choice tests of the Observer

221 Testing phase (OT). Therefore, only 7 individuals of the 17 group members (two males and

222 five females, age range: 14 months to 20 years, mean $=7.4$ years) participated in the

223 experiment (see table 1). One individual (LNG) participated only in the three experimental

224 blocks of the pink condition, again, due motivational reasons. The remaining six individuals

225 completed all six experimental blocks.

\subsection{Experimental Design}

227 We carried out six consecutive experimental blocks, three for the pink condition (P1, P2, P3)

228 and three for the blue condition (B1, B2, B3) (fig. 1). Each experimental block consisted of

229 three distinct phases: (1) Demonstrator Training phase (DT): demonstrators were given the

230 choice between two artificially coloured foods (pink or blue) one of which was rendered

231 unpalatable; (2) Preference Demonstration phase (PD): subjects observed demonstrators

232 choosing their preferred food colours from a distance ranging from approximately $180 \mathrm{~cm}$,

233 across the corridor, to $500 \mathrm{~cm}$ on nearby structures or tunnels; (3) Observer Testing phase

234 (OT) : subjects were given the choice between palatable pink or blue food (courgette or egg,

235 see fig 1). For detailed explanations see below. In the pink condition (blocks P1, P2 and P3)

236 demonstrator(s) were presented with pink and blue courgette, and demonstrator preference

237 was for pink courgette. In the blue condition (blocks B1, B2 and B3) the demonstrator was

238 presented with pink and blue egg and demonstrator preference was for blue egg. 


\begin{tabular}{|c|c|c|c|c|c|c|c|c|}
\hline & \multicolumn{2}{|c|}{$\begin{array}{l}\text { I. Initial } \\
\text { preference }\end{array}$} & \multicolumn{2}{|c|}{$\begin{array}{l}\text { II. New } \\
\text { preference }\end{array}$} & \multicolumn{4}{|c|}{ III. Maintained preference } \\
\hline KEL & $3 \mathrm{PD}$ & OT & $\begin{array}{l}\text { B1 } \\
6 \mathrm{PD}\end{array}$ & $\begin{array}{l}\text { OT } \\
\times 5\end{array}$ & $\begin{array}{ll}\text { B2 } & \\
& 4 \text { PD }\end{array}$ & $\begin{array}{l}\text { OT } \\
\times 5\end{array}$ & $\begin{array}{ll} & \\
& 4 \text { PD }\end{array}$ & $\begin{array}{l}\text { OT } \\
\mathbf{x} 10\end{array}$ \\
\hline DW & $3 \mathrm{PD}$ & $\times 5$ & & & $4 \mathrm{PD}$ & $\begin{array}{l}\text { OT } \\
\times 5\end{array}$ & P3 PD & $\begin{array}{l}\text { OT } \\
\mathbf{x} 10\end{array}$ \\
\hline
\end{tabular}

240 Figure 1. Experimental design - Experimental block order, demonstrator(s) identity, number of

241 Preference Demonstration (PD) days, food used and preferred colour as well as number of Observer

242 Test trials (OT) following each Preference Demonstration phase (PD). Each experimental block began

243 with a Demonstrator Training phase (DT) consisting of 10 food presentations each, not represented on

244 this figure. Testing period for each experimental block in chronological order: P1: Feb, B1: Jul, B2:

245 Aug, B3: Sep, P2: Nov, P3: Nov.

\subsubsection{Demonstrator Training Phase (DT)}

247 In the initial Demonstrator Training phase (DT) for the first experimental block (P1), the two

248 demonstrators, DW and KEL, learned that pink courgette was palatable (artificially

249 sweetened), whereas blue courgette was unpalatable (artificially made bitter). In the three

250 following experimental blocks (B1, B2, B3), KEL then learned the reverse colour pattern,

251 albeit with a different food type (i.e., egg). Training for this new preference was achieved by

252 presenting palatable blue egg, and unpalatable pink egg. Finally, in the last two experimental

253 blocks (P2, P3) DW was provided with refresher training for a maintained preference for pink

254 over blue courgette. In total, DW underwent 30 trials of food preference training with the

255 pink courgette, while KEL underwent 10 with the pink courgette and 30 with the blue egg.

256 To this end, at the start of each experimental block, the demonstrators were visually isolated

257 from the rest of the group (Fig. 2a - cage 8, location marked DT) and were offered the choice

258 between the pink and blue food (courgette or egg depending on the experimental block, see

259 fig. 1 for details), one sweet, one bitter. Food presentation lasted 10 seconds after a first

260 choice had been made. The first choice was defined as the first food touched, although in all 
261 cases this was also the first food item eaten. Both individuals were given the choice 10 times,

262 although we found that the colour-taste association was learned after just one experience with

263 the bitter food. From the next trial onwards, both individuals consistently chose the sweet

264 colour first and either ignored or only cautiously tasted and discarded the bitter colour

265 second. Demonstrator Training (DT) was recorded using a PANASONIC HC-V727 full HD

266 camera equipped with a SENNHEISER MKE 400 external microphone.

267

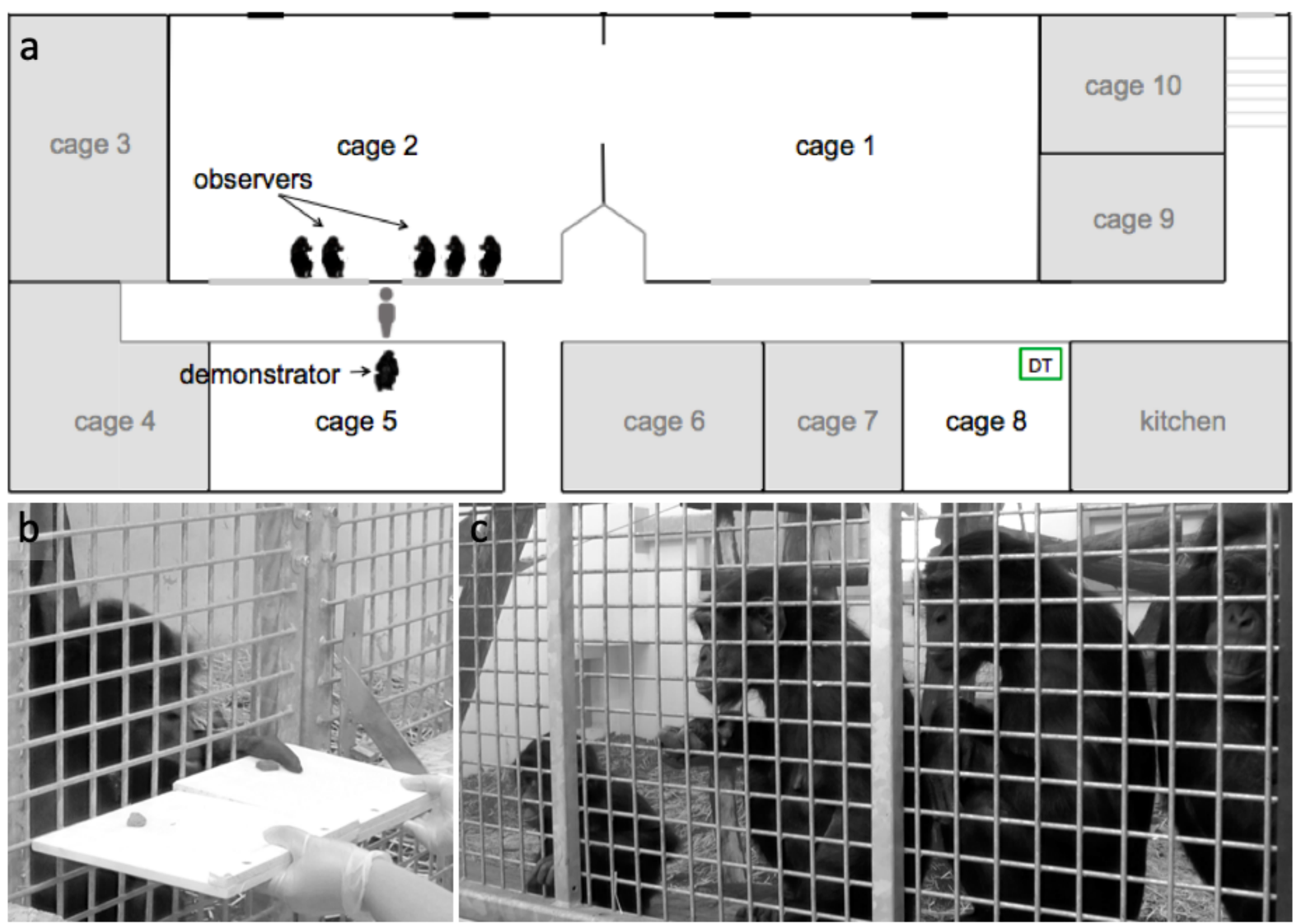

269 Figure 2. Experimental setup for social learning experiment - a) The Preference Demonstration phase

270 (PD) is illustrated showing the demonstrator in cage 5 and the observers across the corridor in cage 2.

271 DT marks the location used in cage 8 for the Demonstrator Training phase; b) Demonstrator choice,

272 manual food presentation using $20 \times 20 \mathrm{~cm}$ white plastic trays; c) Observers watching a Preference

273 Demonstration (PD) from cage 2. 
275 Following the Demonstrator Training phase (DT), a demonstrator was isolated and given the

276 choice between the pink and blue food (Fig. 2b), while the rest of the group observed his

277 choices from the opposite cage (Fig. 2a - cages 5 and 2 and fig. 2c). Demonstrators were

278 allowed to choose 10 times. Food presentation lasted 10 seconds after a first choice had been

279 made, so that observers could see more clearly which food had been chosen and which had

280 been rejected. Preference Demonstrations (PD) were carried out once a day over several

281 consecutive days (see fig. 1 for details).

283 We first ran experimental block P1, in which both DW and KEL were demonstrators and

284 both showed a clear preference for the pink courgette. We chose to start with both

285 demonstrators showing the same preference to increase the salience and strength of the 286 demonstrated preference for the observers. Once established, we proceeded to experimental

287 block B1 in which KEL was sole demonstrator and his colour preference was switched, albeit

288 with a novel food type, so that his preference was for blue egg. We then continued in the blue

289 condition with experimental blocks B2 and B3, again with KEL demonstrating his preference

290 for blue egg. Finally, we carried out experimental blocks P2 and P3, with DW demonstrating

291 his maintained preference for the pink courgette (see fig. 1). The number of demonstration

292 days varied among experimental blocks (i.e. three consecutive demonstration days for each

293 demonstrator in P1, six consecutive days for the sole demonstrator in B1 and four

294 consecutive demonstration days for the sole demonstrator in B2, B3, P2 and P3 resulting in

$295 \mathrm{~N}=170$ observable food choices for KEL and N=110 observable food choices for DW in 296 total, see fig. 1). 
297 During the Preference Demonstration phase (PD) both the demonstrators and the observers

298 where filmed in order to record both demonstrator colour choice and the number of trials

299 attended to by each observer (demonstrator: PANASONIC HC-V727 full HD camera

300 equipped with a SENNHEISER MKE 400 external microphone; observers: PANASONIC

301 HC-V100 full HD and PANASONIC HC-V727 full HD cameras). Subject attention to

302 demonstrator food choice (i.e., defined as the subject's head and eyes oriented towards the

303 demonstrator whilst the choice was made, regardless of posture) was coded post-hoc by GS

304 from the video footage and from oral commentaries recorded during the demonstrations.

305 Subject attention for a given trial was coded conservatively as either ' 1 ' or ' 0 ' (i.e., $1=$

306 observing, $0=$ not observing). Individuals observing from outside the camera range were

307 recorded with help from a trained animal keeper.

\subsubsection{Observer Testing Phase (OT)}

309 Following the Preference Demonstration phase (PD) for each experimental block, observers

310 underwent individual preference testing in which sweetened pink and blue foods (courgette

311 or egg) were presented to them simultaneously. For the Observer Testing phase (OT), both

312 food types were prepared in the same way, but liquid sugarcane was used for all food to rule

313 out any possibility of odour cue-based choices by the observers. Food presentation ended as

314 soon as a choice had been made and was carried out opportunistically on all participating

315 observers $(\mathrm{N}=7)$ in all of the indoor cages and the outdoor enclosure.

317 Observers were tested five times each in blocks P1, P2, B1 and B2, and 10 times each in

318 blocks P3 and B3. Testing was opportunistic but no two consecutive tests were carried out on

319 a given individual unless the individual had resumed another activity before being retested. In

320 order to avoid feeding competition with other group members, the default protocol was to test 
321 subjects in the absence of other group members. It was, however, unavoidable that some subjects ( 5 of 7) witnessed at least one choice test of another subject. In terms of their overall exposure, this was an insignificantly small proportion with far less influence than the

324 Preference Demonstrations (PD) observed (see supplementary material, table S1). Testing was carried out over two or three consecutive days following the Preference Demonstration phase (PD) and was filmed using a PANASONIC HC-V100 full HD camera in order to record the colour chosen. As choices were always unambiguous and clear, we did not carry out any inter-observer reliability tests.

\subsection{Food Preparation and Presentation}

330 Two food types, raw courgettes and cooked egg whites (hereafter 'egg'), were used

331 throughout the experiment. Individuals were familiar with the natural taste of these foods. For

332 all members of the group, courgette is a lesser-valued food; egg is highly valued, but only

333 familiar as hard-boiled and in the shell. For the first two phases of a given experimental block

334 (i.e., Demonstrator Training (DT) and Preference Demonstration (PD)), the two food types were altered in taste and colour to obtain sweet pink courgette and bitter blue courgette for the pink condition, and sweet blue egg and bitter pink egg for the blue condition. We alternated the colour associated with the palatable food to control for any natural colour bias.

338 The courgette was sliced and quartered (size approximately $2 \times 2 \times 0.5 \mathrm{~cm}$ ) before being 339 soaked overnight in either pink food colouring and a sweet additive (liquid sugarcane), or

340 blue food colouring and a bitter additive (Bitrex ${ }^{\circledR}$ aqueous solution $2.5 \%, 0.2 \mathrm{ml}$ per $5 \mathrm{cl}$ of

341 water: 100ppm). For the egg, either blue food colouring and a sweet additive or pink food

342 colouring and a bitter additive were added before cooking the mixture in a microwave oven

343 and cutting it into small pieces (approximately $2 \times 2 \times 0.5 \mathrm{~cm}$ ). For the Observer Testing

344 phase (OT) the two food types were altered in taste and colour to obtain only sweet pink and 
345 blue foods and no bitter foods. Pink and blue food colouring where selected as few to no

346 foods of these colours are included in the group's regular diet. We chose not to carry out a

347 control for a natural colour bias as doing so would have provided the subjects with unwanted

348 experience with the two food colours.

350 To determine preferred food choice, items were offered manually. To this end, the experimenter (GS) placed a cube of each colour of a given food type on two identical white plastic trays $(20 \times 20 \mathrm{~cm})$, which were placed, side by side, against the bars of the cages allowing the individual to reach for the food using either the fingers or lips (Fig. 2b). The

354 position (left or right) of the different coloured food items was balanced and pseudo-

355 randomised, insofar as no given colour was presented on the same side for no more than three consecutive trials.

\subsection{Statistical Analyses}

358 Statistical analyses were carried out using R 3.1.2., GUI 1.65 and SPSS version 22. Datasets

359 are available online [https://doi.org/10.17632/tczyxb9wvh.1]. Wilcoxon exact sign rank tests

360 were used to test comparisons between: 1) Subject attention to the two demonstrators (i.e.,

361 the percentage of DW and KEL Preference Demonstration (PD) trials observed by each

362 subject); 2) Performance in the first choice test, (i.e. number of matched and non-matched

363 choices made in the first test following each Preference Demonstration (PD)). For the overall

364 performance (i.e., the number of matched and non-matched choices made by each subject

365 overall), we ran a Generalised linear model with a quasi-binomial error structure. We

366 modelled the probability of making matched choice across all trials per individual as a

367 function of age and proportion of observed Preference Demonstration (PD) trials. 
3.1 Preference Demonstration Phase (PD)

\subsubsection{Demonstrator performance}

371 KEL and DW's performance during Preference Demonstrations (PD) was close to perfect.

372 They chose the correct colour first in $97 \%$ and $99 \%$ of trials and ate the correct colour

373 exclusively in $95 \%$ and $96 \%$ of trials, respectively (i.e. pink courgette in P1, P2 and P3 and

374 blue egg in B1, B2 and B3; KEL N=170 and DW N=110, see Preference Demonstration 375 phase (PD) in methods for details).

\subsubsection{Attention to demonstrators}

377 While observers paid more attention to Preference Demonstrations (PD) by DW than to those 378 by KEL, the difference was not statistically significant (mean percentage of demonstrations watched by the subjects: DW 35.4\%, KEL $29.8 \%$, Wilcoxon exact test, $\mathrm{Z}=-1.014, \mathrm{~N}=7$,

380 exact $P=0.38$, two-tailed).

\subsection{Observer Testing Phase (OT)}

\subsubsection{First choice performance}

383 For the first trial of the first experimental block (P1) all observers were naïve having had no personal experience with the artificially altered foods. Nevertheless, six of seven subjects chose the colour chosen by the demonstrator in this first trial. When analysing first choices across all experimental blocks, individuals chose the matched colour significantly more often than the non-matched colour $(\mathrm{Z}=-2.64, \mathrm{~N}=6, P=0.031$, two-tailed, Wilcoxon exact test; Fig. 3; one subject, LNG, could not be included in this analysis as she partook in only 3 of the 6 experimental blocks). Of the seven subjects, six chose mostly the matched colour as 
390 their first choice for each experimental block, and only one chose the matched and non-

391 matched colour equally, suggesting that, as per our predictions, individuals immediately

392 experienced a significant bias towards the foods chosen by the demonstrators, a clear

393 demonstration of rapid social learning by observation.

394

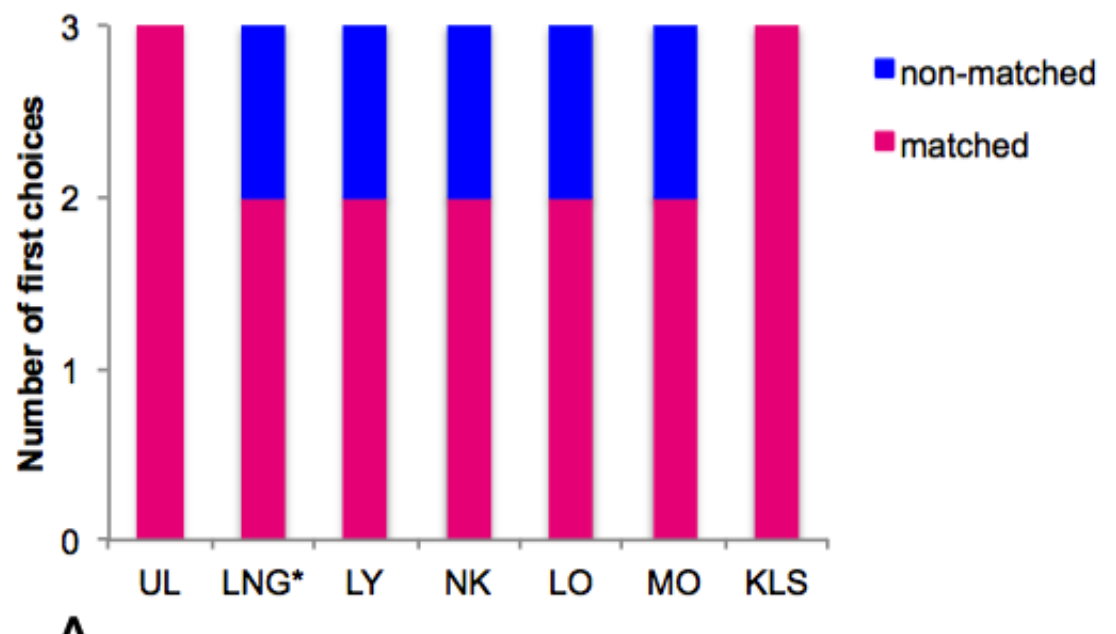

395

A

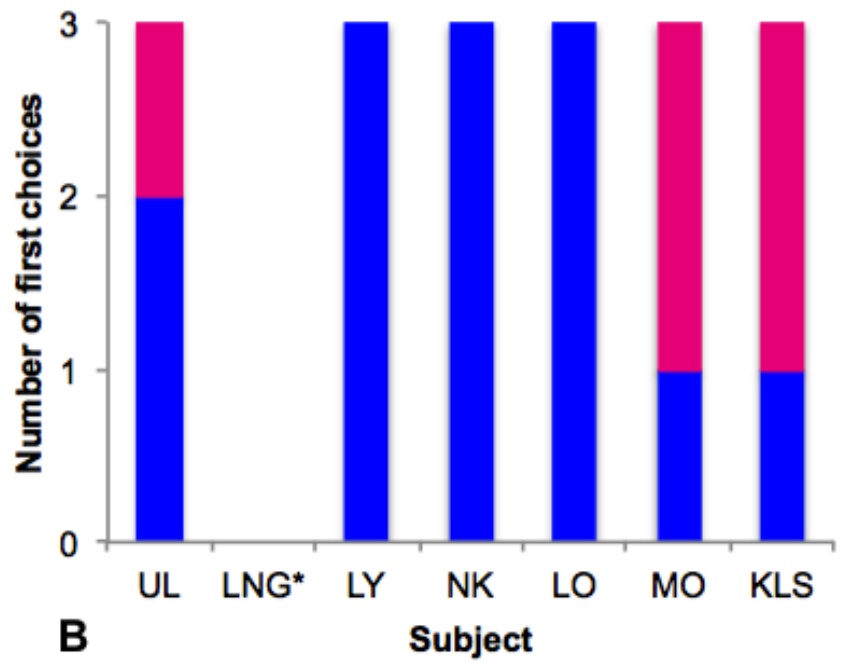

non-matched

matched

396

397 Figure 3. Number of matched and non-matched colour choices for each subject in the first choice test

398 of the Observer Testing phase (OT): A) pink experimental blocks (P1, P2, P3), B) blue experimental

399 blocks (B1, B2, B3), subjects classed by decreasing age from left to right. 
401 Taking into account the observers' overall performance in choosing the matched colour,

402 results show that the food preference of the demonstrators continued to have an influence on

403 observer colour choice. Indeed, the model supported the fact that subjects matched their

404 choices to those of the demonstrators significantly above chance levels (see table 2 and figure

4054 for raw data).

406

407 Table 2. Results of the logistic regression modelling the proportion of matched trials $(\mathrm{N}=7)$.

\begin{tabular}{lllll}
\hline & Estimate & Standard error & Z value & $\mathrm{P}$ \\
\hline Intercept & 0.64 & 0.09 & 6.85 & $0.002 * * *$ \\
Age & 7.33 & 1.60 & 4.59 & $0.010 *$ \\
Proportion of trials observed & 1.65 & 0.50 & 3.32 & $0.029 *$ \\
\hline
\end{tabular}

408 Estimates are on a logit scale.

409 This is important because, over the course of the experiment, all observers occasionally tasted

410 the alternative colour choice (i.e., unmatched choice), which was identical in taste and

411 palatability to the matched choice (both treated with liquid sugarcane). Nonetheless, they

412 chose the food colour preferred by the demonstrators more than the alternative.

413 


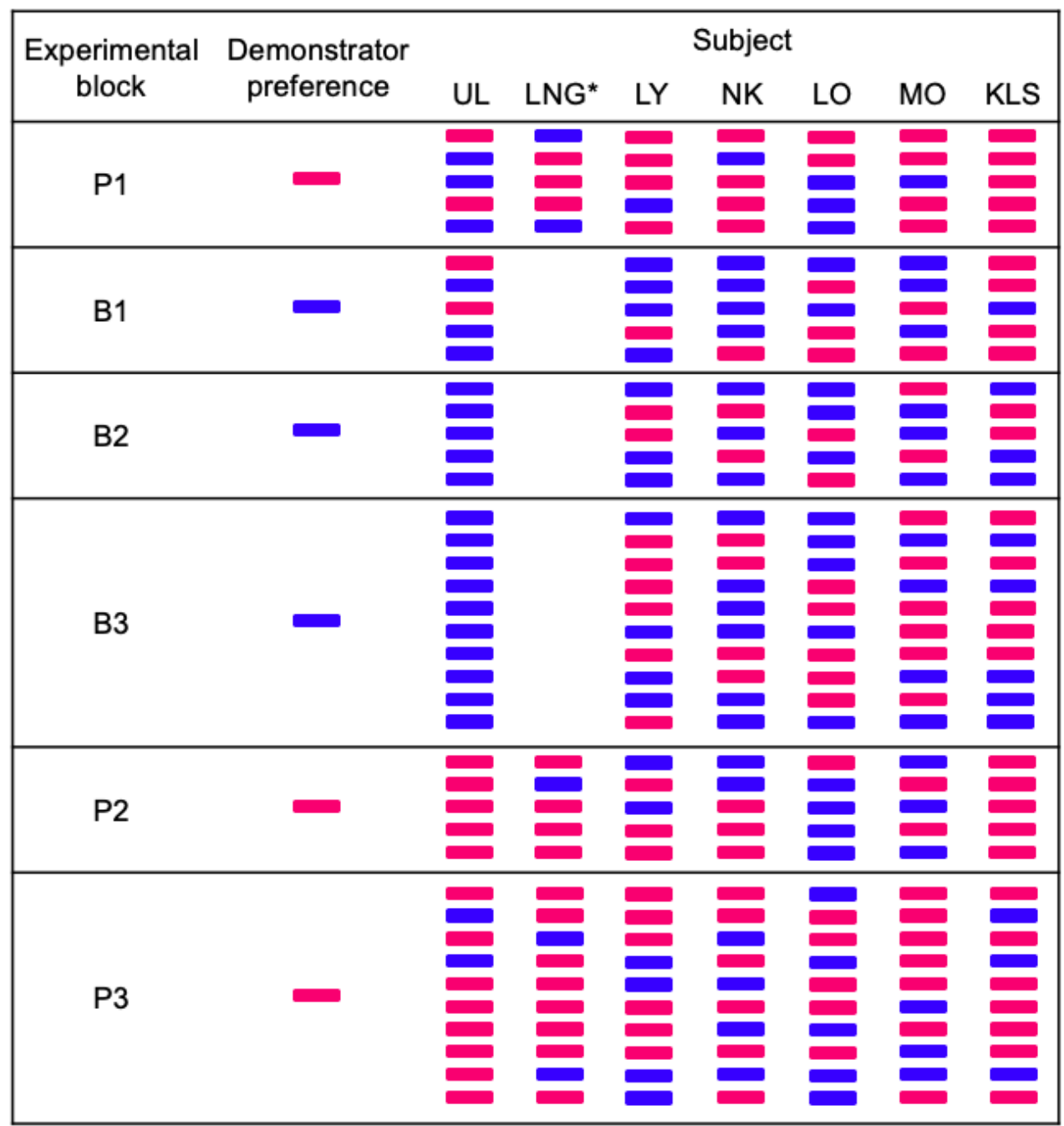

415 Figure 4. Experimental block, demonstrator preference and all subject choices during the Observer

416 Testing phase (OT) for all six experimental blocks (both subject choice trials and experimental blocks 417 are shown in chronological order).

418 Furthermore, both age and proportion of trials observed had a significant positive effect on 419 the proportion of matched choices (Table 2, Figs. 5 and 6). 


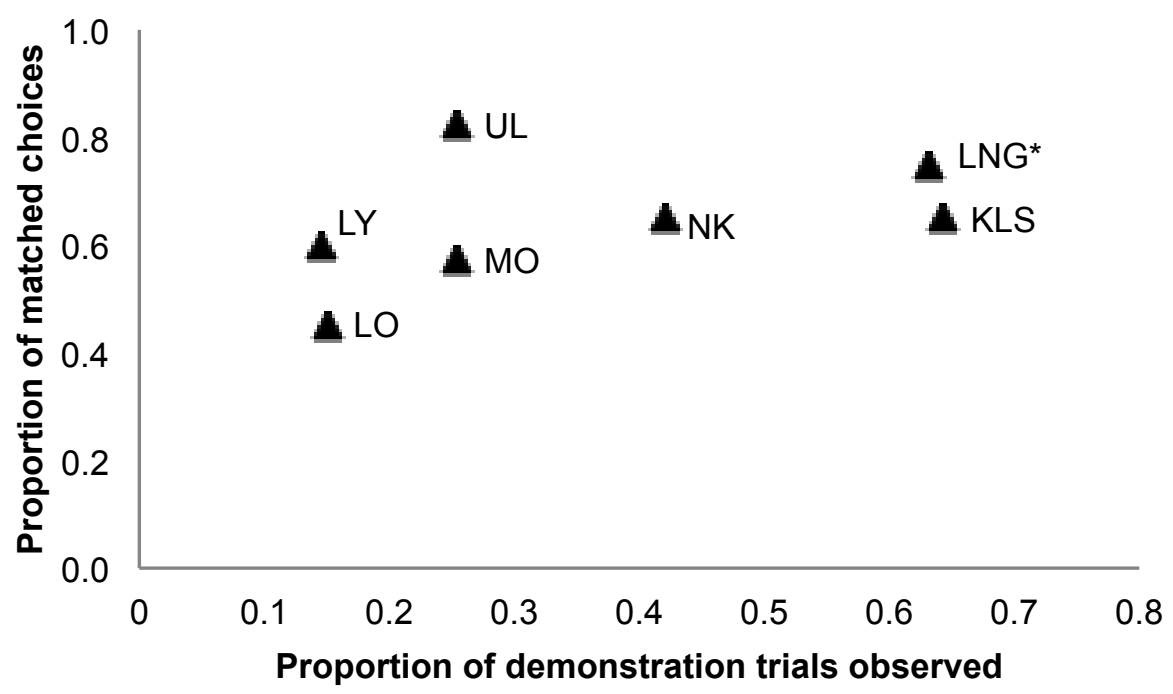

421

422 Figure 5. Proportion of matched choices made during Observer Testing phase (OT) and proportion 423 of Preference Demonstration (PD) trials observed by each subject. LNG, marked by an asterisk, 424 participated in only P1, P2 \& P3.

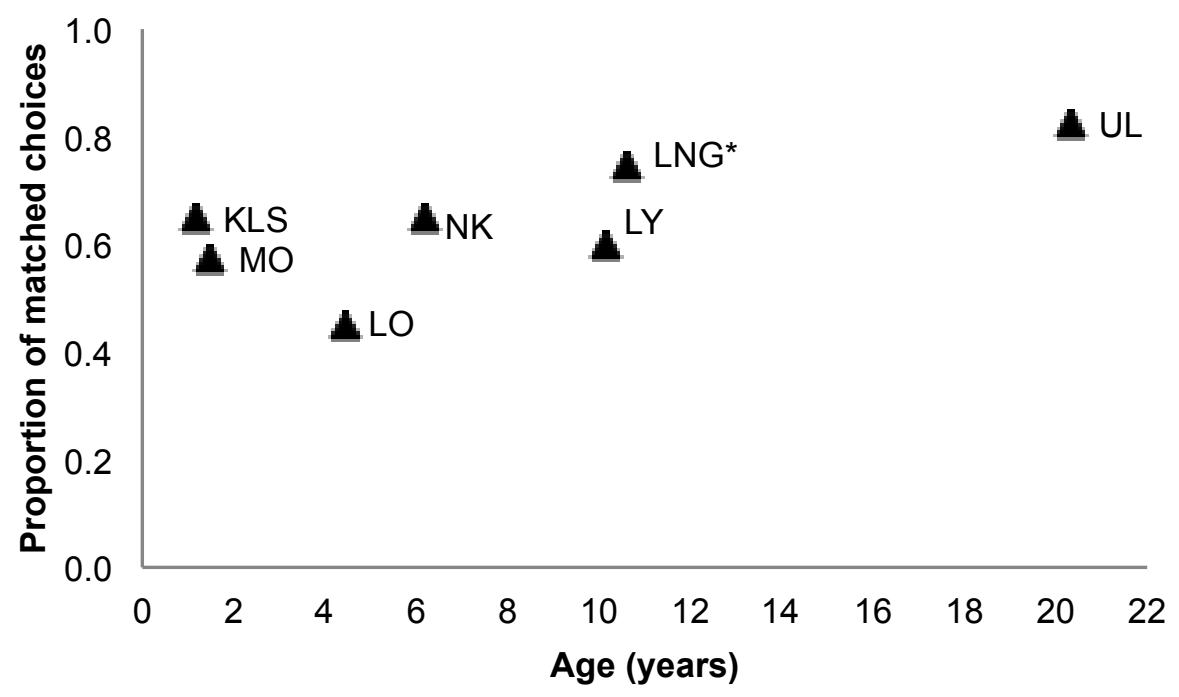

426 Figure 6. Proportion of matched choices made during the Observer Testing phase (OT) and subject 427 age in years. LNG, marked by an asterisk, participated in only P1, P2 \& P3.

429 When considering individual performance (i.e. percentage of matched choices during the 430 Observer Testing phase (OT)), it can be noted that there is considerable inter-individual 431 variability (Table 3), variability which can be explained in part by the age of subjects and 
their attention to Preference Demonstrations (PD) (as shown in the model). Nonetheless, six of seven subjects chose the matched food colour more often than the unmatched food colour.

Table 3. Individual performance over all six experimental blocks

\begin{tabular}{lcccc}
\hline Subject & $N$ & $\begin{array}{c}\text { Proportion of demonstration } \\
\text { trials observed }\end{array}$ & $\begin{array}{c}\text { Food colour choice } \\
\text { matched : unmatched }\end{array}$ & $\begin{array}{c}\text { Performance } \\
\text { (\% matched })\end{array}$ \\
\hline UL & 40 & 0.25 & $33: 7$ & 82.5 \\
LNG & 20 & 0.63 & $15: 5$ & 75.0 \\
LY & 40 & 0.14 & $24: 16$ & 60.0 \\
NK & 40 & 0.42 & $26: 14$ & 65.0 \\
LO & 40 & 0.15 & $18: 22$ & 45.0 \\
MO & 40 & 0.25 & $23: 17$ & 57.5 \\
KLS & 40 & 0.64 & $26: 14$ & 65.0 \\
\hline
\end{tabular}

436 Proportion of demonstration trials observed, number of matched and unmatched choices and

437 percentage of matched choices made by subjects. One individual (LNG) participated in the Observer 438 Testing phase (OT) for only three of the six experimental blocks: P1, P2 \& P3.

\section{Discussion}

440 In this study we sought to investigate whether bonobos are capable of acquiring and

441 retaining information relating to arbitrary food preferences of fellow group members

442 through mere observation. In other words, can bonobos socially learn the different food

443 preferences of other group members and do they abide to these even if doing so goes against

444 their own experience and knowledge (i.e., despite knowing that both colour alternatives are

445 equally palatable)? In our experiment, both demonstrators were exposed to trial-and-error

446 learning to install an arbitrary preference for visually novel food items, which led

447 immediately to a clear bias for the palatable food. In a series of six experimental blocks,

448 subjects were then provided with the opportunity to observe demonstrator preferences before 
being presented with the choice of two colour alternatives of an otherwise identical food item. The results, as demonstrated by the number of matched choices made by the subjects in the first choice of each experimental block, showed that the subjects had developed an immediate preference for the food item chosen by the demonstrator, demonstrating rapid and reliable social learning. Furthermore, and importantly, this effect occurred despite subjects being exposed to a complex demonstration pattern during which one demonstrator (KEL) demonstrated opposing colour preferences for different foods (see fig. 1). In this paradigm which confronted the subjects with the question of "what to eat?" it is likely that the mechanism at work is simple stimulus enhancement as is often the case with this and other everyday behavioural questions such as "where to sleep?" or "who and what to beware of?"

459 (Whiten and van de Waal, 2018).

Our second finding relates to the fact that subjects' performance continued to be biased towards the demonstrated preference, as indicated in the subjects' overall performance, even after individuals had opportunities to taste the alternatively coloured foods (i.e., when both coloured foods were known to be equally palatable, see fig. 4 for frequencies of alternative colour choice throughout the experiment). We noted considerable inter-individual differences in performance during the crucial Observer Testing phase (OT), which was at least partially explained by subject age and exposure to Preference Demonstrations (PD) (see fig. 5 and 6); indeed, younger individuals and those that observed only a few demonstrations were less likely to match their choices to those of the demonstrators in the Observer Testing phase (OT) than older individuals or those that observed many demonstrations. It seems quite intuitive that performance should improve with increased exposure to demonstrator preference, however, the result observed in relation to subject age is in contradiction with the general finding that suggests that juvenile primates are more 
prone to use social information than are adults (e.g. Coelho et al., 2015; Matsuzawa, 1994).

475

476

477

478

479

480

481

482

483

484

485

486

487

488

489

490

491

492

493

494

495

496

497

498

In the present study we found that older subjects were those most likely to match their choices to those of the demonstrators.

When considering the possible explanations for subjects generally adhering to demonstrator preferences we must consider the recent findings which have revealed that rather than being set rules, social learning strategies are used flexibly at the individual, group and population level (see Kendal et al., 2018 for a non-exhaustive review of theoretical and empirical support for a broad range of social learning strategies), in fact a recent study in the wild has demonstrated that this flexibility can even be at the species level (Bono et al., 2018). In their review, Kendal et al. (2018) present a variety of strategies as to when, whom and what to copy. In our study, following their initial choice, not only were subjects rapidly exposed to the alternative colour choice, but in choosing the alternative colour they were immediately rewarded, just as they were when copying the demonstrator. And yet in our study, subjects generally adhered to the preference shown by the demonstrator. This propensity to adhere to demonstrator preference might be in line with a 'when' learning strategy more expected of naïve subjects: 'copy-when-uncertain'. This would suggest that subjects perceived uncertainty during testing which increased their willingness to rely on social information (Galef et al., 2008; Kendal et al., 2015) rather than their own experience. In some studies with apes and human children subjects have been found to follow the demonstrator's strategy even if this meant going against their own personal preference (e.g. human children, Gergely et al., 2002; chimpanzees, Hopper et al., 2011) while in other studies this was not the case (Vale et al., 2017). Vale and colleagues (2017), for instance, demonstrated that chimpanzees preferred to rely on their personal experience with unpalatable foods rather than to conform to group norms. Observation of group members eating the previously 'unpalatable' food did, 
however, promote the re-exploration of that food through social learning. This pattern of relying more on one's own experience than on social information from conspecifics has also

501 been observed in other animal species. For example, Fryday and Greig-Smith (1994),

502 demonstrated that the amount of food consumed by house sparrows was influenced not by

503 demonstrator consumption, but by their own previous experience with the food (i.e., palatable untreated food or unpalatable quinine-treated food).

Regarding a possible "who" strategy, there is much empirical work to support model-based biases, for instance the propensity to learn from older and higher ranking group members (e.g. Biro et al., 2003; Kendal et al., 2015). In our study, the two demonstrators were not the highest ranking male in the group, nor were they the oldest, and neither one was directly related to any of the subjects, nevertheless social learning took place, as shown, for example,

511 by the results obtained for UL, a high-ranking adult female, who was most strongly

512 influenced by these males (i.e., UL made the highest number of matched choices of all

513 subjects, see table 3 and fig. 4). This result may be indicative of a 'who' bias for

514 'knowledgeable' or 'expert' models (Whiten and van de Waal, 2018) or perhaps, similarly to chimpanzees, in the absence of 'demonstrator-competition' (i.e., the presence of the usually more favoured, older and higher-ranking demonstrators), low-ranking individuals are able to

517 successfully seed a behaviour (Watson et al., 2017). This finding is thus at odds with one of

518 de Waal's (2001) bonding- and identification-based observational learning model predictions,

519 which is that, for social learning to take place, demonstrators ought to be high ranking 520 individuals, a pattern that has been found in both captive (Horner et al., 2010) and wild

521 chimpanzees (Hobaiter et al., 2014) although kin-based learning may be more important in 522 establishing long-term behavioural traditions (Lamon et al., 2017). 
523 Kendal et al. further present a set of frequency-dependant biases. Indeed, potential

524 explanation for subjects adhering to demonstrator preference, despite possessing the

525 knowledge that both foods were equally palatable, might come from some sort of desire to

526 conform (as predicted by de Waal's (2001) BIOL model, although the author predicted a

527 desire to conform specifically to higher ranking and older individuals), perhaps for the sake

528 of social cohesion (Hopper et al., 2011). In humans, this effect is particularly strong if a novel

529 behaviour is shown by several or a majority of group members. Conformity predicts that

530 individuals will change their own behaviour and adopt the majority behaviour in order to

531 comply with what they perceive as 'social norms' (van Leeuwen and Haun, 2014). Whether

532 or not such social influence really requires a majority (e.g. Asch, 1956) or comes into play

533 even when the behaviour has initially been demonstrated by a minority, is often unclear

534 (Hopper et al., 2011; but see Cialdini and Trost, 1984 for a review). In our study we did not

535 test specifically for conformity, since subjects did not have to adhere to the behaviour of a

536 majority, instead we demonstrated that several group members adopted the same preference

537 (non-exclusively) as that expressed by the two demonstrators, and this was despite having

538 knowledge that both foods were equally palatable.

539

540 Another explanation is that subjects were following a sensible survival strategy: if a

541 demonstrator systematically refuses to eat a certain food, then it may be reasonable to assume

542 that he does so for a good reason. In the present case, following the demonstrator's choice

543 and following a 'better-safe-than-sorry' strategy was cost free. The underlying drivers of such

544 behaviour open questions for future research. 
546 We conclude that, even in the absence of olfactory and taste cues, bonobos are able to acquire

547 and memorise others' food preferences and are prepared to adhere to them, even when the

548 demonstrator is not a high ranking individual. Bonobos, similar to humans, are susceptible to

549 acquiring information from watching the behaviour of others even if demonstrators are socially

550 unimportant and even adhere to these learned behaviours despite personal knowledge that there

551 is no additional reward in doing so. Furthermore, while this study does not allow us to draw

552 conclusions regarding the exact social learning mechanism(s) at work, the results, even if due

553 to simple stimulus enhancement, as has been suggested for social learning of many of the day-

554 to-day behaviours (Whiten and van de Waal, 2018), demonstrate that subjects matched their

555 choices to those of the demonstrators regardless of the colour, food type and demonstrator

556 identity. We believe this supports not only the fact that learning from others in the context of

557 food acquisition is of paramount importance but also provides further support to the ongoing

558 discussion around the flexible use of social learning strategies.

559 Finally, it is important to point out that our findings were made in the context of an

560 ecologically important situation, i.e. learning about novel foods, which highlights the fact that

561 social learning theory is likely to benefit from paradigms that focus on more universal

562 ecologically relevant problems.

\section{Acknowledgements}

564 The research leading to these results has received funding from the European Research

565 Council under the European Union's Seventh Framework Programme (FP7/2007-2013)/

566 ERC grant agreement $\mathrm{n}^{\circ} 283871$ and the Swiss National Science Foundation (Social learning

567 in primate communication: 31003A_166458 / Coordinating joint action in apes: Testing the 
568 boundaries of the human interaction engine: CR31I3_159655). We thank "La Vallée des

569 Singes" and its director, Emmanuel Le Grelle, as well as "Le Conservatoire pour la

570 Conservation des Primates" for allowing access to the study site and subjects. We also thank

571 keepers, Carole Michelet, Franck Alexieff, Jérémy Mergault, and Alexandre Albert for their

572 valuable help in carrying out the experiment as well as Josep Call, Redouan Bshary and

573 Christof Neumann for valuable discussion as well as Radu Slobodeanu for statistical advice.

574 Declarations of interest: none 


\section{References}

576

577

578

579

580

581

582

583

584

585

586

587

588

589

590

591

592

593

594

595

596

597

598

599

600

601

602

603

604

605

606

607

608

609

610

611

612

613

614

615

616

617

618

619

620

Addessi, E., Galloway, A.T., Visalberghi, E., Birch, L.L., 2005. Specific social influences on the acceptance of novel foods in 2-5-year-old children. Appetite 45, 264-271. https://doi.org/10.1016/j.appet.2005.07.007

Addessi, E., Visalberghi, E., 2001. Social facilitation of eating novel food in tufted capuchin monkeys (Cebus apella): input provided by group members and responses affected in the observer. Animal Cognition 4, 297-303. https://doi.org/10.1007/s100710100113

Animal Behaviour, 1992. Guidelines for the use of animals in research. Animal Behaviour 43, 185-188.

Aplin, L.M., Farine, D.R., Morand-Ferron, J., Cockburn, A., Thornton, A., Sheldon, B.C., 2014. Experimentally induced innovations lead to persistent culture via conformity in wild birds. Nature 518, 538-541. https://doi.org/10.1038/nature13998

Asch, S.E., 1956. Studies of independance and conformity: I. A minority of one against a unanimous majority. Psychological Monographs 70, 1-70.

Auersperg, A.M.I., von Bayern, A.M.I., Weber, S., Szabadvari, A., Bugnyar, T., Kacelnik, A., 2014. Social transmission of tool use and tool manufacture in Goffin cockatoos (Cacatua goffini). Proceedings of the Royal Society B: Biological Sciences 281, 20140972. https://doi.org/10.1098/rspb.2014.0972

Avarguès-Weber, A., Dawson, E.H., Chittka, L., 2013. Mechanisms of social learning across species boundaries: Social learning across species boundaries. Journal of Zoology 290, 1-11. https://doi.org/10.1111/jzo.12015

Biro, D., Inoue-Nakamura, N., Tonooka, R., Yamakoshi, G., Sousa, C., Matsuzawa, T., 2003. Cultural innovation and transmission of tool use in wild chimpanzees: evidence from field experiments. Animal Cognition 6, 213-223. https://doi.org/10.1007/s10071-0030183-X

Bono, A.E.J., Whiten, A., Van Schaik, C.P., Krützen, M., Eichenberger, F., Schnider, A., van de Waal, E., 2018. Payoff- and sex-biased social learning interact in a wild primate population. Current Biology 28, 2800-2805.e4. https://doi.org/10.1016/j.cub.2018.06.015

Byrne, R.W., Corp, N., Byrne, J.M.E., 2001. Estimating the complexity of animal behaviour: How mountain gorillas eat thistles. Behaviour 138, 525-557. https://doi.org/10.1163/156853901750382142

Call, J., Tomasello, M., 1994. The social learning of tool use by orangutans (Pongo pygmaeus). Human Evolution 9, 297-313.

Cialdini, R.B., Trost, M.R., 1984. Social influence: Social norms, conformity, and compliance, in: Gilbert, D.T., Fiske, S.T., Lindzey, G. (Eds.), The Handbook of Social Psychology. Oxford University Press, Oxford, pp. 151-192.

Clay, Z., Tennie, C., 2018. Is Overimitation a Uniquely Human Phenomenon? Insights From Human Children as Compared to Bonobos. Child Development 89, 1-10. https://doi.org/10.1111/cdev.12857

Coelho, C.G., Falótico, T., Izar, P., Mannu, M., Resende, B.D., Siqueira, J.O., Ottoni, E.B., 2015. Social learning strategies for nut-cracking by tufted capuchin monkeys (Sapajus spp.). Animal Cognition 18, 911-919. https://doi.org/10.1007/s10071-015-0861-5

Coolen, I., Bergen, Y.V., Day, R.L., Laland, K.N., 2003. Species difference in adaptive use of public information in sticklebacks. Proceedings of the Royal Society B: Biological Sciences 270, 2413-2419. https://doi.org/10.1098/rspb.2003.2525 
Coussi-Korbel, S., Fragaszy, D.M., 1995. On the relation between social dynamics and social learning.pdf. Animal Behaviour 50, 1441-1453.

Dawson, E.H., Chittka, L., 2012. Conspecific and Heterospecific Information Use in Bumblebees. PLoS ONE 7, e31444. https://doi.org/10.1371/journal.pone.0031444 de Waal, F.B.M., 2001. The ape and the sushi master: Cultural reflections of a primatologist. Basic Books, New York, NY.

Dindo, M., Stoinski, T., Whiten, A., 2011. Observational learning in orangutan cultural transmission chains. Biol. Lett. 7, 181-183. https://doi.org/10.1098/rsbl.2010.0637

Dindo, M., Thierry, B., Whiten, A., 2008. Social diffusion of novel foraging methods in brown capuchin monkeys (Cebus apella). Proceedings of the Royal Society B: Biological Sciences 275, 187-193. https://doi.org/10.1098/rspb.2007.1318

Dindo, M., Whiten, A., de Waal, F.B.M., 2009. In-group conformity sustains different foraging traditions in capuchin monkeys (Cebus apella). PLoS ONE 4, e7858. https://doi.org/10.1371/journal.pone.0007858

Fairbanks, L., 1975. Communication of food quality in captive Macaca nemestrina and freeranging Ateles geoffroyi. Primates 16, 181-190.

Finestone, E., Bonnie, K.E., Hopper, L.M., Vreeman, V.M., Lonsdorf, E.V., Ross, S.R., 2014. The interplay between individual, social, and environmental influences on chimpanzee food choices. Behavioural Processes 105, 71-78. https://doi.org/10.1016/j.beproc.2014.03.006

Fryday, S.L., Greig-Smith, P.W., 1994. The effects of social learning on the food choice of the house sparrow (Passer domesticus). Behaviour 128, 281-300.

Galef, B.G., Dudley, K.E., Whiskin, E.E., 2008. Social learning of food preferences in 'dissatisfied' and 'uncertain' Norway rats. Animal Behaviour 75, 631-637. https://doi.org/10.1016/j.anbehav.2007.06.024

Galef, B.G., Giraldeau, L.-A., 2001. Social influences on foraging in vertebrates: causal mechanisms and adaptive functions. Animal Behaviour 61, 3-15. https://doi.org/10.1006/anbe.2000.1557

Galef, B.G., Laland, K.N., 2005. Social learning in animals: empirical studies and theoretical models. Bioscience 55, 489-499.

Gergely, G., Bekkering, H., Király, I., 2002. Developmental psychology: Rational imitation in preverbal infants. Nature 415, 755-755.

Gustafsson, E., Saint Jalme, M., Bomsel, M.-C., Krief, S., 2014. Food Neophobia and Social Learning Opportunities in Great Apes. International Journal of Primatology 35, 10371071. https://doi.org/10.1007/s10764-014-9796-y

Heyes, C.M., 1994. Social learning in animals: categories and mechanisms. Biological Reviews 69, 207-231.

Hobaiter, C., Poisot, T., Zuberbühler, K., Hoppitt, W., Gruber, T., 2014. Social network analysis shows direct evidence for social transmission of tool use in wild chimpanzees. PLoS Biology 12, e1001960. https://doi.org/10.1371/journal.pbio.1001960

Hopper, L.M., Schapiro, S.J., Lambeth, S.P., Brosnan, S.F., 2011. Chimpanzees' socially maintained food preferences indicate both conservatism and conformity. Animal Behaviour 81, 1195-1202. https://doi.org/10.1016/j.anbehav.2011.03.002

Horner, V., Proctor, D., Bonnie, K.E., Whiten, A., de Waal, F.B.M., 2010. Prestige affects cultural learning in chimpanzees. PLoS ONE 5, e10625. https://doi.org/10.1371/journal.pone.0010625

Horner, V., Whiten, A., 2005. Causal knowledge and imitation/emulation switching in chimpanzees (Pan troglodytes) and children (Homo sapiens). Animal Cognition 8, 164-181. https://doi.org/10.1007/s10071-004-0239-6 
686

687

688

689

690

691

692

693

694

695

696

697

698

699

700

701

702

703

704

705

706

707

708

709

710

711

712

713

714

715

716

Huffman, M.A., Hirata, S., 2004. An experimental study of leaf swallowing in captive chimpanzees: insights into the origin of a self-medicative behavior and the role of social learning. Primates 45, 113-118. https://doi.org/10.1007/s10329-003-0065-5

Hunt, G.R., Gray, R.D., Taylor, A.H., 2013. Why is tool use rare in animals?, in: Sanz, C.M., Call, J., Boesch, C. (Eds.), Tool Use in Animals: Cognition and Ecology. Cambridge University Press, Cambridge, pp. 89-118.

Jaeggi, A.V., Dunkel, L.P., Van Noordwijk, M.A., Wich, S.A., Sura, A.A.L., Van Schaik, C.P., 2010. Social learning of diet and foraging skills by wild immature Bornean orangutans: implications for culture. American Journal of Primatology 72, 62-71. https://doi.org/10.1002/ajp.20752

Kano, T., 1984. Distribution of pygmy chimpanzees (Pan paniscus) in the central Zaire basin. Folia primatologica 43, 36-52.

Kawai, M., 1965. Newly-acquired pre-cultural behavior of the natural troop of Japanese monkeys on Koshima Islet. Primates 6, 1-30.

Kendal, R., Hopper, L.M., Whiten, A., Brosnan, S.F., Lambeth, S.P., Schapiro, S.J., Hoppitt, W., 2015. Chimpanzees copy dominant and knowledgeable individuals: implications for cultural diversity. Evolution and Human Behavior 36, 65-72. https://doi.org/10.1016/j.evolhumbehav.2014.09.002

Kendal, R.L., Boogert, N.J., Rendell, L., Laland, K.N., Webster, M., Jones, P.L., 2018. Social Learning Strategies: Bridge-Building between Fields. Trends in Cognitive Sciences 22, 651-665. https://doi.org/10.1016/j.tics.2018.04.003

Kitchen, D.M., Bergman, T.J., Cheney, D.L., Nicholson, J.R., Seyfarth, R.M., 2010. Comparing responses of four ungulate species to playbacks of baboon alarm calls. Animal Cognition 13, 861-870. https://doi.org/10.1007/s10071-010-0334-9

Koops, K., Furuichi, T., Hashimoto, C., 2015. Chimpanzees and bonobos differ in intrinsic motivation for tool use. Scientific Reports 5. https://doi.org/10.1038/srep11356

Laland, K.N., 2004. Social Learning Strategies. Learning and Behaviour 32, 4-14.

Lamon, N., Neumann, C., Gruber, T., Zuberbühler, K., 2017. Kin-based cultural transmission of tool use in wild chimpanzees. Science Advances 3, e1602750.

Lamon, N., Zuberbühler, K., 2015. Object manipulation and tool use in wild chimpanzees of the Budongo forest, Uganda. Folia Primatologica 86, 235-386. https://doi.org/10.1159/000435825

Marshall-Pescini, S., Whiten, A., 2008. Social learning of nut-cracking behavior in East African sanctuary-living chimpanzees (Pan troglodytes schweinfurthii). Journal of Comparative Psychology 122, 186-194. https://doi.org/10.1037/0735-7036.122.2.186

Mason, J.R., Reidinger, R.F., 1981. Effects of social facilitation and observational learning on feeding behaviour of the red-winged blackbird (Agelaius phoeniceus). The Auk 98, 778-784.

Matsuzawa, T., 1994. Field experiments on use of stone tools by chimpanzees in the wild, in: Wrangham, R.W., McGrew, W.C., De Waal, F.B.M., Heltne, P.G. (Eds.), Chimpanzee Cultures. Harvard University Press, Cambridge, MA, US, pp. 351-370.

Menzel, C., Fowler, A., Tennie, C., Call, J., 2013. Leaf Surface Roughness Elicits Leaf Swallowing Behavior in Captive Chimpanzees (Pan troglodytes) and Bonobos (P. paniscus), but not in Gorillas (Gorilla gorilla) or Orangutans (Pongo abelii). International Journal of Primatology 34, 533-553. https://doi.org/10.1007/s10764013-9679-7

Rapaport, L.G., Brown, G.R., 2008. Social influences on foraging behavior in young nonhuman primates: Learning what, where, and how to eat. Evolutionary Anthropology: Issues, News, and Reviews 17, 189-201. https://doi.org/10.1002/evan.20180 
Reader, S.M., Biro, D., 2010. Experimental identification of social learning in wild animals. Learning \& Behavior 38, 265-283. https://doi.org/10.3758/LB.38.3.265

Rendell, L., Fogarty, L., Hoppitt, W.J.E., Morgan, T.J.H., Webster, M.M., Laland, K.N., 2011. Cognitive culture: theoretical and empirical insights into social learning strategies. Trends in Cognitive Sciences 15, 68-76. https://doi.org/10.1016/j.tics.2010.12.002

Reynolds, V., 2005. The Chimpanzees of the Budongo Forest: Ecology, behaviour and conservation. Oxford University Press, Oxford.

Samuni, L., Mundry, R., Terkel, J., Zuberbühler, K., Hobaiter, C., 2014. Socially learned habituation to human observers in wild chimpanzees. Animal Cognition 17, 9971005. https://doi.org/10.1007/s10071-014-0731-6

Seppänen, J.-T., Forsman, J.T., 2007. Interspecific Social Learning: Novel Preference Can Be Acquired from a Competing Species. Current Biology 17, 1248-1252. https://doi.org/10.1016/j.cub.2007.06.034

Snowdon, C.T., Boe, C.Y., 2003. Social communication about unpalatable foods in tamarins (Saguinus oedipus). Journal of Comparative Psychology 117, 142-148. https://doi.org/10.1037/0735-7036.117.2.142

Stoinski, T.S., Drayton, L.A., Price, E.E., 2011. Evidence of social learning in black-andwhite ruffed lemurs (Varecia variegata). Biology Letters 7, 376-379. https://doi.org/10.1098/rsbl.2010.1070

Tarnaud, L., 2004. Ontogeny of feeding behavior of Eulemur fulvus in the dry forest of Mayotte. International Journal of Primatology 25, 803-824.

Tennie, C., Hedwig, D., Call, J., Tomasello, M., 2008. An experimental study of nettle feeding in captive gorillas. American Journal of Primatology 70, 584-593. https://doi.org/10.1002/ajp.20532

Ueno, A., Matsuzawa, T., 2005. Response to novel food in infant chimpanzees. Behavioural Processes 68, 85-90. https://doi.org/10.1016/j.beproc.2004.09.002

Vale, G.L., Davis, S.J., van de Waal, E., Schapiro, S.J., Lambeth, S.P., Whiten, A., 2017. Lack of conformity to new local dietary preferences in migrating captive chimpanzees. Animal Behaviour 124, 135-144. https://doi.org/10.1016/j.anbehav.2016.12.007

van de Waal, E., Borgeaud, C., Whiten, A., 2013a. Potent social learning and conformity shape a wild primate's foraging decisions. Science 340, 483-485. https://doi.org/10.1126/science.1232769

van de Waal, E., Bshary, R., Whiten, A., 2014. Wild vervet monkey infants acquire the foodprocessing variants of their mothers. Animal Behaviour 90, 41-45. https://doi.org/10.1016/j.anbehav.2014.01.015

van de Waal, E., Claidière, N., Whiten, A., 2013b. Social learning and spread of alternative means of opening an artificial fruit in four groups of vervet monkeys. Animal Behaviour 85, 71-76. https://doi.org/10.1016/j.anbehav.2012.10.008

van de Waal, E., Renevey, N., Favre, C.M., Bshary, R., 2010. Selective attention to philopatric models causes directed social learning in wild vervet monkeys. Proceedings of the Royal Society B: Biological Sciences 277, 2105-2111. https://doi.org/10.1098/rspb.2009.2260

van Leeuwen, E.J.C., Haun, D.B.M., 2014. Conformity without majority? The case for demarcating social from majority influences. Animal Behaviour 96, 187-194. http://dx.doi.org/10.1016/j.anbehav.2014.08.004

Visalberghi, E., Addessi, E., 2000. Response to change in food palatability in tufted capuchin monkeys, Cebu apella. Animal Behaviour 59, 231-238. 
Visalberghi, E., Sirianni, G., Fragaszy, D., Boesch, C., 2015. Percussive tool use by Taï Western chimpanzees and Fazenda Boa Vista bearded capuchin monkeys: a comparison. Philosophical Transactions of the Royal Society B: Biological Sciences 370, 20140351. https://doi.org/10.1098/rstb.2014.0351

Vitousek, M.N., Adelman, J.S., Gregory, N.C., Clair, J.J.H.S., 2007. Heterospecific alarm call recognition in a non-vocal reptile. Biology Letters 3, 632-634. https://doi.org/10.1098/rsbl.2007.0443

Watson, S.K., Reamer, L.A., Mareno, M.C., Vale, G., Harrison, R.A., Lambeth, S.P., Schapiro, S.J., Whiten, A., 2017. Socially transmitted diffusion of a novel behavior from subordinate chimpanzees. American Journal of Primatology 79, e22642. https://doi.org/10.1002/ajp.22642

Whitehead, J.M., 1986. Development of feeding selectivity in mantled howling monkeys, Alouatta palliata, in: Else, J.G., Lee, P.C. (Eds.), Primate Ontogeny, Cognition and Social Behaviour. Cambridge University Press, Cambridge, pp. 105-117.

Whiten, A., 2005. The second inheritance system of chimpanzees and humans. Nature 437 , 52-55. https://doi.org/10.1038/nature04023

Whiten, A., Custance, D.M., Gomez, J.-C., Teixidor, P., Bard, K.A., 1996. Imitative learning of artificial fruit processing in children (Homo sapiens) and chimpanzees (Pan troglodytes). Journal of comparative psychology 110, 3.

Whiten, A., Horner, V., de Waal, F.B.M., 2005. Conformity to cultural norms of tool use in chimpanzees. Nature 437, 737-740. https://doi.org/10.1038/nature04047

Whiten, A., Mesoudi, A., 2008. Establishing an experimental science of culture: animal social diffusion experiments. Philosophical Transactions of the Royal Society B: Biological Sciences 363, 3477-3488. https://doi.org/10.1098/rstb.2008.0134

Whiten, A., van de Waal, E., 2018. The pervasive role of social learning in primate lifetime development. Behavioral Ecology and Sociobiology 72. https://doi.org/10.1007/s00265-018-2489-3

Whiten, A., van de Waal, E., 2016a. Social learning, culture and the 'socio-cultural brain' of human and non-human primates. Neuroscience \& Biobehavioral Reviews. https://doi.org/10.1016/j.neubiorev.2016.12.018

Whiten, A., van de Waal, E., 2016b. Identifying and dissecting conformity in animals in the wild: further analysis of primate data. Animal Behaviour 122, e1-e4. https://doi.org/10.1016/j.anbehav.2016.09.010

Yamakoshi, G., 1998. Dietary responses to fruit scarcity of wild chimpanzees at Bossou, Guinea: Possible implications for ecological importance of tool use. American Journal of Physical Anthropology 106, 283-295. https://doi.org/10.1002/(SICI)10968644(199807)106:3<283::AID-AJPA2>3.0.CO;2-O

Zuberbühler, K., 2000. Interspecies semantic communication in two forest primates. Proceedings of the Royal Society of London. Series B: Biological Sciences 267, 713718. 


\begin{tabular}{|c|c|c|c|c|c|c|c|c|}
\hline 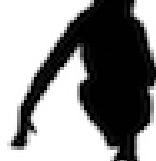 & \multicolumn{2}{|c|}{$\begin{array}{c}\text { I. Initial } \\
\text { preference }\end{array}$} & \multicolumn{2}{|c|}{$\begin{array}{c}\text { II. New } \\
\text { preference }\end{array}$} & \multicolumn{4}{|c|}{ III. Maintained preference } \\
\hline KEL & $\mathrm{P1}$ & 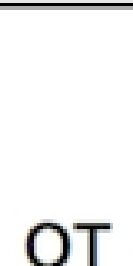 & $\begin{array}{ll} & \\
& \\
& 6 \text { PD }\end{array}$ & $\begin{array}{l}\text { OT } \\
\times 5\end{array}$ & $\begin{array}{ll} & \\
& \\
& 4 \text { PD }\end{array}$ & $\begin{array}{l}\text { OT } \\
\times 5\end{array}$ & $\begin{array}{l}\text { B3 } \\
4 \text { PD }\end{array}$ & $\begin{array}{l}\text { OT } \\
\times 10\end{array}$ \\
\hline DW & $3 \mathrm{PD}$ & $\times 5$ & & & P2 PD & $\begin{array}{l}\text { OT } \\
\times 5\end{array}$ & P3 PD & $\begin{array}{l}\text { OT } \\
\times 10\end{array}$ \\
\hline
\end{tabular}




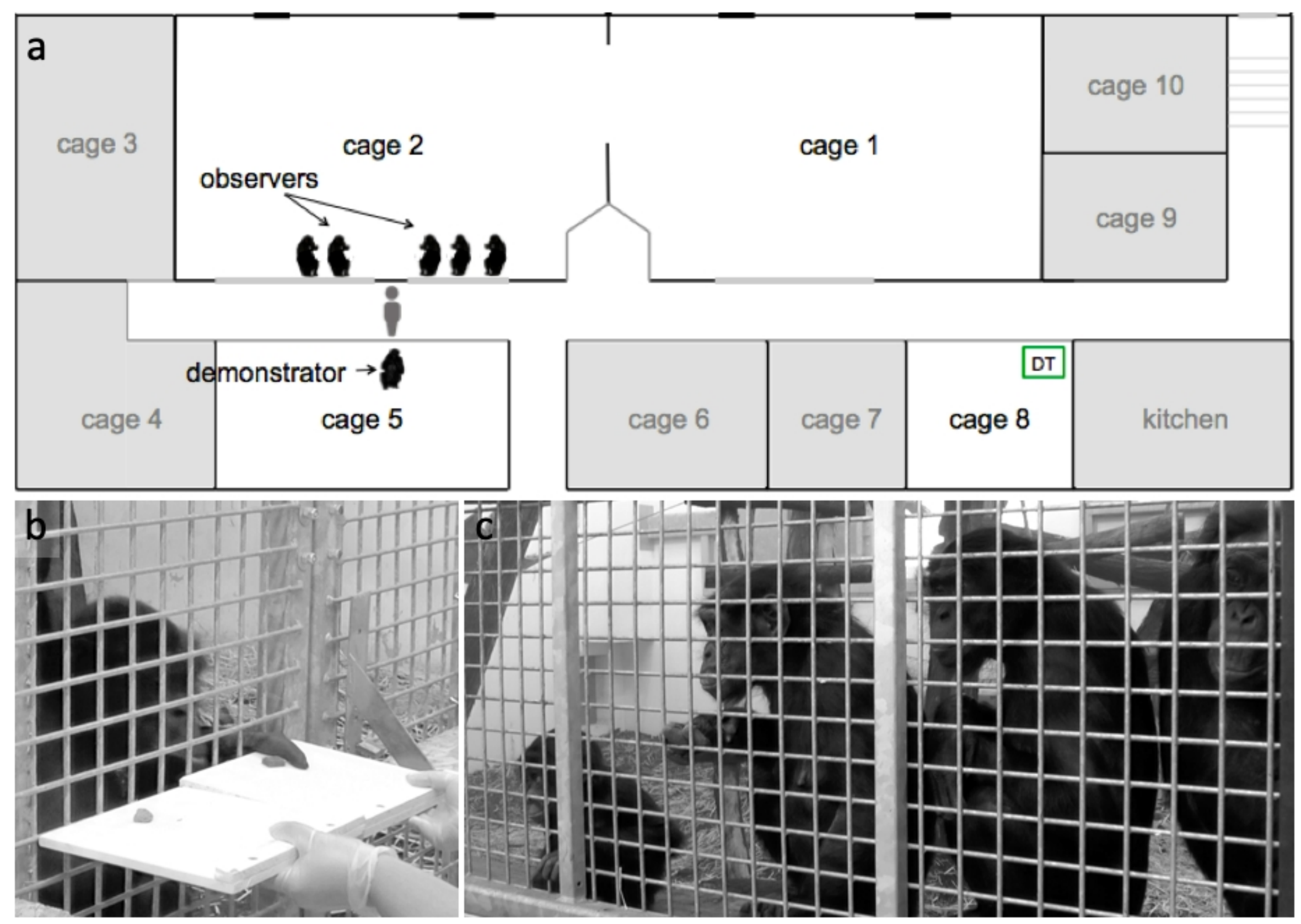




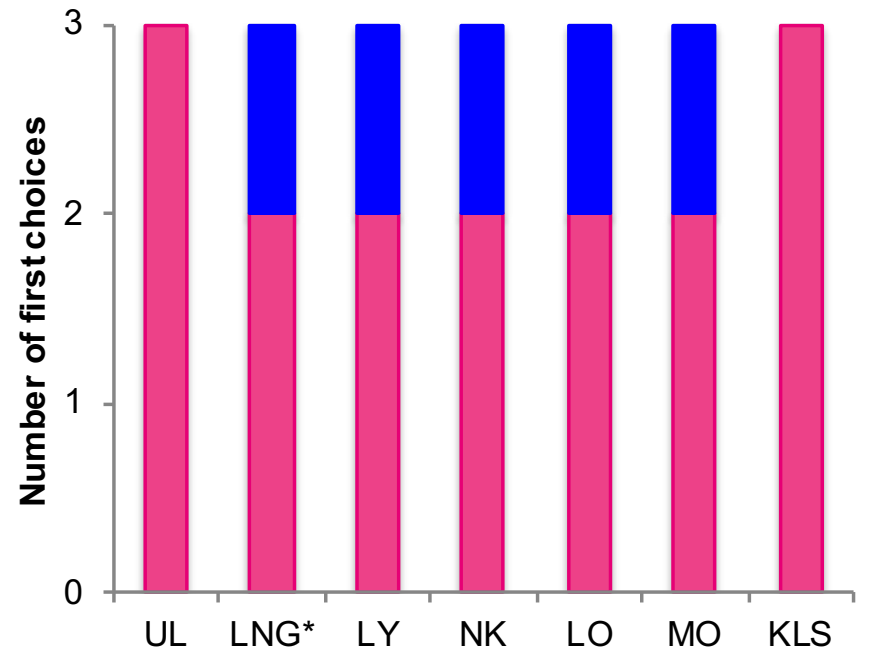

non-matched

a matched 


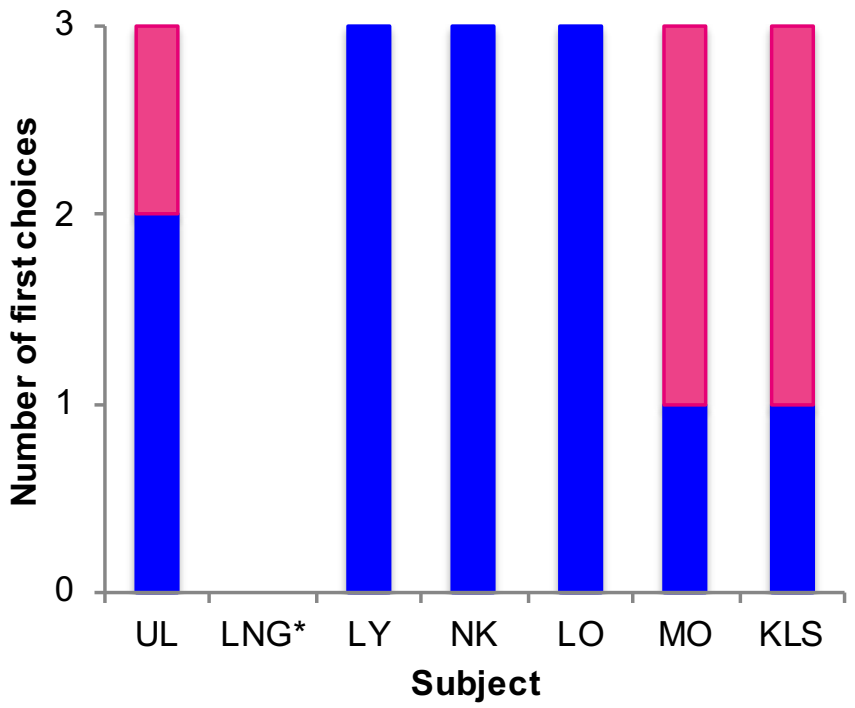

anon-matched

matched 
Experimental Demonstrator

Subject

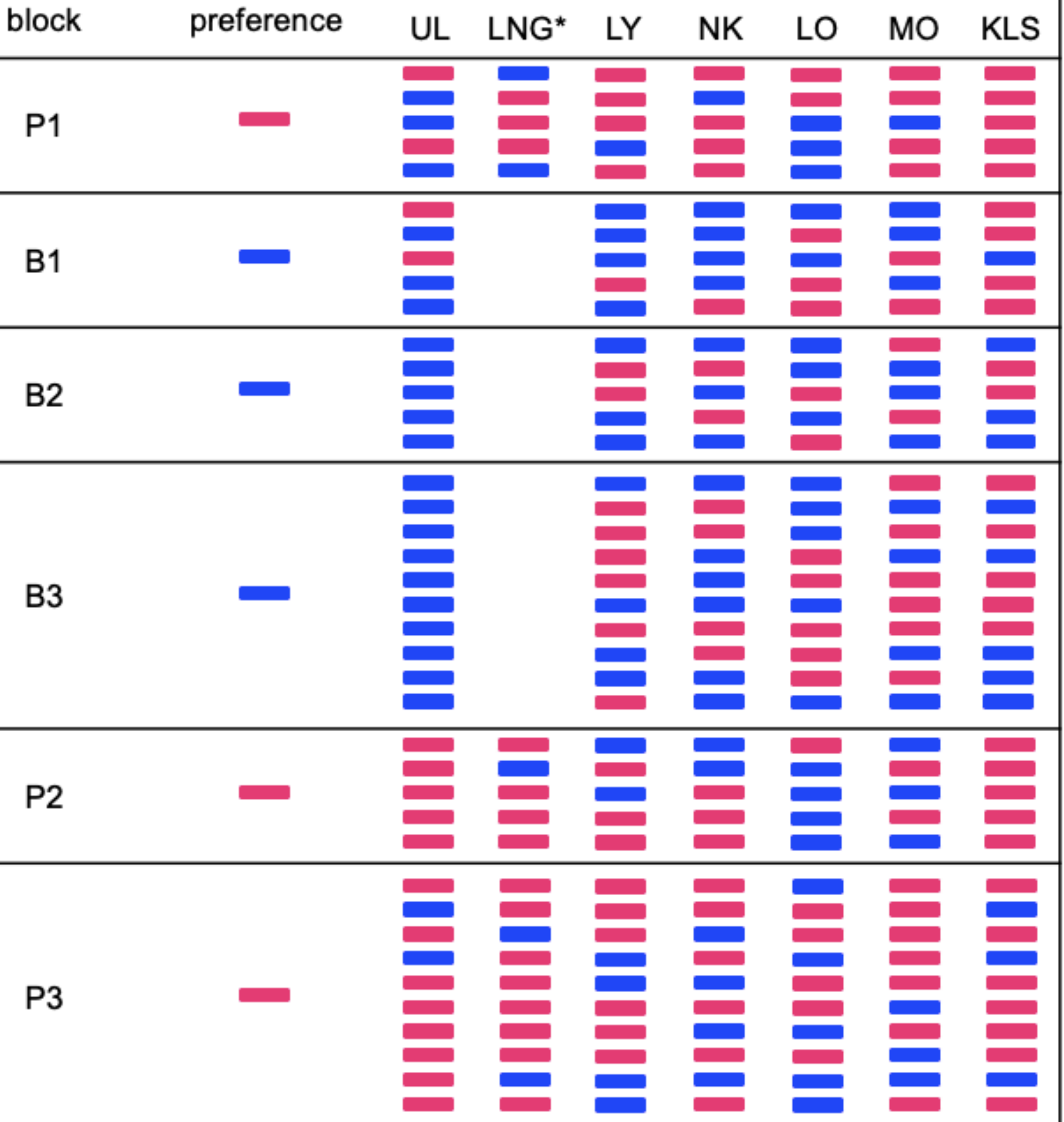




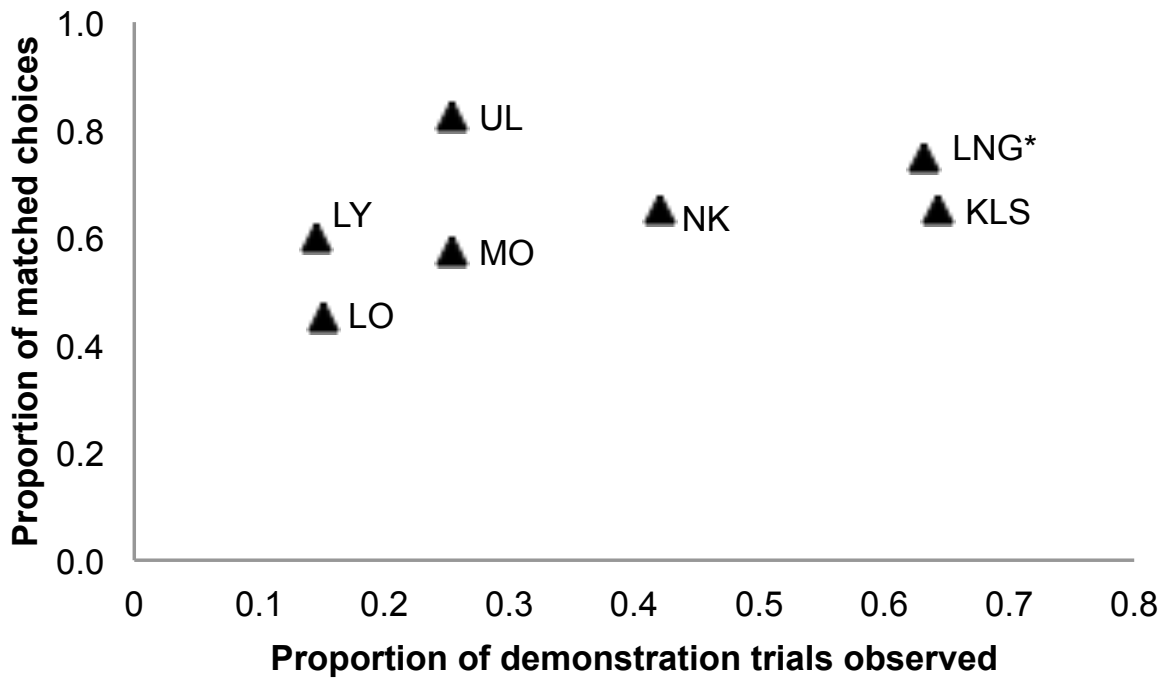




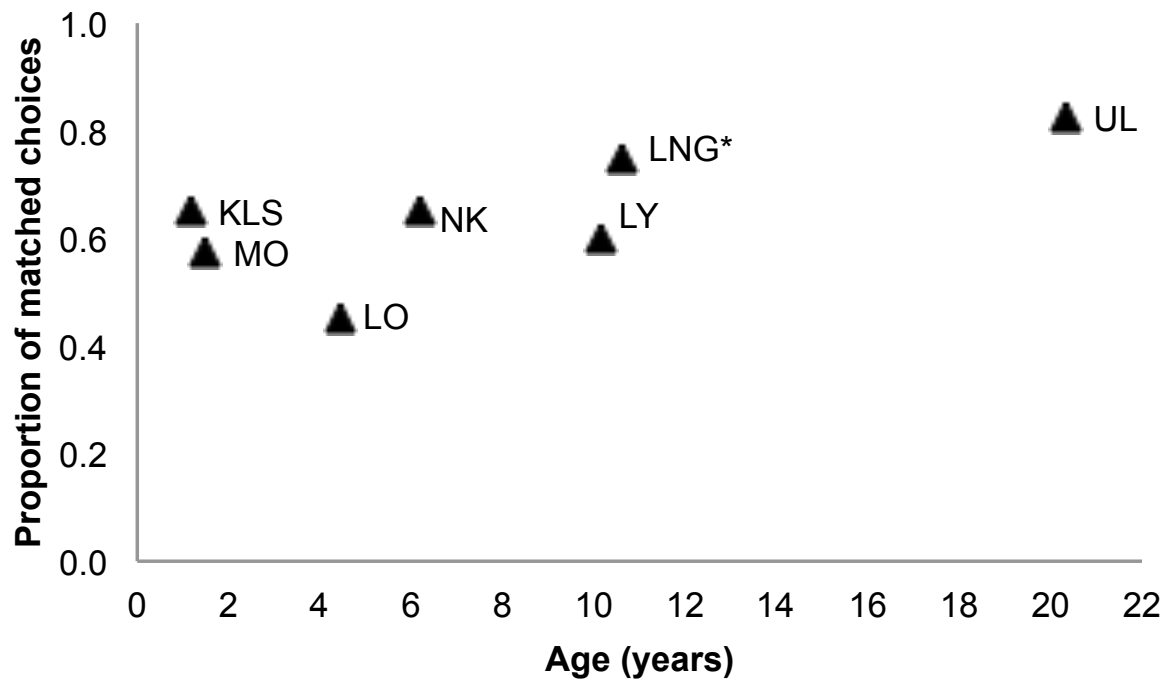


Table 1. Study subjects housed at La Vallée des Singes and role in the experiment

\begin{tabular}{llllll}
\hline \multicolumn{2}{l}{ Individual } & Sex & Birth year & Age-class & Role \\
\hline Diwani & $(\mathrm{DW})$ & $\mathrm{M}$ & 1996 & Adult & Demonstrator \\
Kelele & $(\mathrm{KEL})^{*}$ & $\mathrm{M}$ & 2004 & Adult & Demonstrator \\
\hline Ulindi & $(\mathrm{UL})$ & $\mathrm{F}$ & 1993 & Adult & Observer \\
Lingala & $(\mathrm{LNG})$ & $\mathrm{F}$ & 2003 & Sub-adult & Observer \\
Lucy & $(\mathrm{LY})$ & $\mathrm{F}$ & 2003 & Sub-adult & Observer \\
Nakala & $(\mathrm{NK})^{*}$ & $\mathrm{~F}$ & 2007 & Juvenile & Observer \\
Loto & $(\mathrm{LO})$ & $\mathrm{M}$ & 2009 & Juvenile & Observer \\
Moko & $(\mathrm{MO})$ & $\mathrm{M}$ & 2012 & Infant & Observer \\
Khalessi & $(\mathrm{KLS})$ & $\mathrm{F}$ & 2012 & Infant & Observer \\
\hline
\end{tabular}

Individuals marked by an asterisk had the same father; age-class as defined by Kano (1984) 
Table 2. Results of the logistic regression modelling the proportion of matched trials $(\mathrm{N}=7)$.

\begin{tabular}{lllll}
\hline & Estimate & Standard error & Z value & $\mathrm{P}$ \\
\hline Intercept & 0.64 & 0.09 & 6.85 & $0.002 * * *$ \\
Age & 7.33 & 1.60 & 4.59 & $0.010 *$ \\
Proportion of trials observed & 1.65 & 0.50 & 3.32 & $0.029 *$ \\
\hline
\end{tabular}

Estimates are on a logit scale. 
Table 3. Individual performance over all six experimental blocks

\begin{tabular}{lcccc}
\hline Subject & $N$ & $\begin{array}{c}\text { Proportion of demonstration } \\
\text { trials observed }\end{array}$ & $\begin{array}{c}\text { Food colour choice } \\
\text { matched : unmatched }\end{array}$ & $\begin{array}{c}\text { Performance } \\
\text { (\% matched) }\end{array}$ \\
\hline UL & 40 & 0.25 & $33: 7$ & 82.5 \\
LNG & 20 & 0.63 & $15: 5$ & 75.0 \\
LY & 40 & 0.14 & $24: 16$ & 60.0 \\
NK & 40 & 0.42 & $26: 14$ & 65.0 \\
LO & 40 & 0.15 & $18: 22$ & 45.0 \\
MO & 40 & 0.25 & $23: 17$ & 57.5 \\
KLS & 40 & 0.64 & $26: 14$ & 65.0 \\
\hline
\end{tabular}

Proportion of demonstration trials observed, number of matched and unmatched choices and percentage of matched choices made by subjects. One individual (LNG) participated in the Observer Testing phase (OT) for only three of the six experimental blocks: P1, P2 \& P3. 